\title{
Los Balleneros y el conocimiento de los mares del sur en la primera parte del siglo XIX
}

\section{Whalers and the Knowledge of the South Seas in the First Half of the 19th Century}

SUSANA V. GARCÍA*

Resumen La industria ballenera constituyó un negocio a gran escala que generó diversos documentos escritos. Sus agentes tuvieron una gran movilidad, operando en puertos distantes y regiones no mapeadas e interactuando con diferentes espacios naturales y tradiciones culturales. En este trabajo se examinan los tipos de registro y observaciones producidas por esta actividad en los mares del sur durante la primera parte del siglo XIX y las maneras en que esa información circuló, se organizó y fue utilizada entre sectores científicos y navales. Focalizando en la explotación pelágica y la navegación a vela se busca demostrar que la información sobre recursos explotables y descubrimiento de islas y buenos fondeaderos, circuló por amplias redes conformadas por nodos de encuentro y comunicación como los barcos, los muelles y los periódicos comerciales. A partir del análisis de diferentes fuentes históricas, se revisan algunas cuestiones para pensar de qué forma la cultura marítima del mundo ballenero ayudó a modelar un conocimiento global de los océanos.

Palabras-clave actividad ballenera, registros escritos, diarios de navegación

\footnotetext{
* https://orcid.org/0000-0001-5151-9230

CONICET, Museo de La Plata, UNLP

Paseo del Bosque, s/n, B1904CMC, Buenos Aires, Argentina

garcia_su@yahoo.com.ar
} 
Aвstract The whaling industry was a large-scale business that generated a variety of written documents. Its agents were highly mobile, operating in distant ports and unmapped regions and interacting with different natural spaces and cultural traditions. This paper examines the types of records and observations produced by this activity in the South Seas during the first part of the nineteenth century and the ways in which this information circulated, was organised and used among scientific and naval sectors. Focusing on pelagic exploitation and sailing, the aim is to demonstrate that information on exploitable resources and the discovery of islands and good anchorages circulated through wide networks made up of meeting and communication nodes such as ships, wharfs and commercial newspapers. From the analysis of different historical sources, some questions are reviewed in order to think about how the maritime culture of the whaling world helped to shape a global knowledge of the oceans.

KEYwORDS Whaling, Written Records, Ship's Logbooks

Pensar en los océanos como espacios de producción del conocimiento implica prestar atención a las prácticas y saberes de trabajadores marítimos especializados como capitanes, pilotos, pescadores, balleneros y loberos, quienes por distintas vías intervinieron en la recopilación y circulación de información y en las discusiones sobre la naturaleza de esos espacios. En el caso de los agentes vinculados a la explotación comercial de mamíferos marinos, estos tuvieron en general una gran movilidad, operando en puertos distantes y en regiones no mapeadas e interactuando con mundos y tradiciones muy diferentes. Esta industria movió la economía y pesó en la política internacional, impulsando la expansión territorial de varios países y la identificación de nuevos espacios de explotación mercantil. Los balleneros y loberos definieron nuevas rutas de navegación, de comercio y de contrabando, trasegando datos y objetos, relacionando océanos, islas, continentes, imperios y remotos lugares. Como se ha mencionado frecuentemente en la amplia historiografía 
ballenera, en el siglo XIX contribuyeron con los conocimientos geográficos e hidrográficos, especialmente con el descubrimiento de islas en el Océano Pacífico y en los mares antárticos, mientras sus diarios de viaje sirvieron para mapear la distribución mundial de cetáceos. Algunos de estos capitanes también prestarían colaboración en las expediciones navales y proveerían de dibujos y objetos a los naturalistas. Este trabajo propone examinar los tipos de registro y observaciones producidas por este tipo de actividad en la primera parte del siglo XIX y las maneras en que esa información circuló, se organizó y fue utilizada entre sectores científicos y navales en el conocimiento de la naturaleza oceánica. En ese sentido, se procura revisar algunas cuestiones para pensar de qué forma la cultura marítima del mundo ballenero ayudó a modelar un conocimiento global de los océanos.

Tradicionalmente muchas de las historias sobre las actividades balleneras se han construido como parte de una historiografía nacional o local, aunque desde hace unos años esa tendencia se está revirtiendo con propuestas de estudios comparativos y el análisis de las diversas experiencias de explotación ballenera en Sudamérica (QUIROZ; TOLEDO, 2014; CASTELLUCCI JUNIOR, 2015; COMERLATO; QUIROZ, 2019; QUIROZ, 2020). Este trabajo se suma a estas propuestas que abren nuevas preguntas y temas sobre la historia ballenera. Focalizando en la explotación pelágica y la navegación a vela en los inicios del siglo XIX, en las siguientes páginas se busca demostrar que la información sobre recursos explotables y descubrimiento de islas y buenos fondeaderos, circuló entre los barcos y muelles sin respetar nacionalidades ni banderas como también ocurrió con la movilidad y las actividades de sus agentes. En ese sentido, los documentos relevados muestran que los empresarios balleneros y armadores compilaron informes y datos de sus capitanes, de sus socios y sus representantes comerciales en puertos de diferentes países. Indudablemente, la industria ballenera se movió por los océanos y el papel. Ello puede ser pensado en términos de las llamadas tecnologías del papel y de una historia burocrática del conocimiento (PODGORNY, 2018; 2019), es decir, en función del análisis de 
los protocolos y los medios que organizan la información, conectando la historia de la ciencia con la historia de la explotación de recursos de origen animal, la cultura de la burocracia mercantil y la práctica de los registros náuticos.

\section{LA “PESCA DEL SUR” Y LOS REGISTROS ESCRITOS}

En el último cuarto del siglo XVIII, se organiza la llamada "pesca del sur" desde los puertos del Atlántico norte, una antítesis de la explotación comercial de cetáceos y pinnípedos en los mares del Norte (JENKINS, 1921; JACKSON, 1978; DU PASQUIER, 1982; 1990; DICKINSON, 2007). Por entonces, el gran consumo de los productos obtenidos de estos animales y la rentabilidad de este comercio impulsaron la expansión de esta actividad cinegética desde una escala regional a una global, propagándose por el Atlántico hacia el sur del Ecuador en la década de 1770 y luego por el Océano Indico y el Pacífico hacia 1790 (RICHARDS, 1994). Lugares de "pesca" distantes implicaron largas travesías, mayores inversiones de capital, una mejor planificación de los viajes y del diseño de las embarcaciones para poder realizar las operaciones pelágicas lejos de los puertos de partida. La actividad ballenera se constituyó en un negocio a gran escala que generó diversos documentos escritos.

Entre 1775 y 1800 se refinó el equipamiento ballenero, las prácticas mercantiles ligadas a esta industria y el conocimiento sobre los patrones migratorios de las ballenas francas del sur, los lugares de abundancia de cachalotes y otros animales marinos como elefantes de mar, lobos marinos y pingüinos, cuyos huevos se aprendieron a conservar para la alimentación en las largas travesías. Según Richards (1994), esas travesías para "pescar" en los llamados "bancos de Brasil"1 sirvió a los balleneros para testear e implementar las tecnologías y los productos para una industria ballenera global que se mantuvo prácticamente sin cambios por casi un siglo. También se consolidó un grupo de expertos

1 Cabe señalar que en las bahías de Brasil se practicaba la explotación costera de ballenas desde el siglo XVII (ELLIS, 1968; CASTELLUCCI JÚNIOR, 2010; EDMUNDSON; HART, 2014). 
en estas operaciones: armadores, capitanes, arponeros de la isla de Nantucket y otros puertos de Nueva Inglaterra, varios de los cuales se vincularon con la "pesca del sur" organizada desde distintos puertos británicos y francesas (CLAYTON, 2007; DU PASQUIER, 1982; SINEUX, 1986). Tras finalizar las guerras napoleónicas y restablecerse la paz en los mares, al menos 81 capitanes de Nantucket comandaron barcos balleneros franceses hacia los mares del sur y 149 capitanearon barcos británicos (CATALOGUE, 1876).

Las operaciones y los lugares de la "gran pesca" se propagaron rápidamente a través de la contratación de estos agentes y personal especializados de Nueva Inglaterra, unidos en gran parte por lazos familiares y religiosos. A ello se sumó los consejos y las noticias que circularon por los espacios de sociabilidad marítima: ya sea en los muelles balleneros y sus fondas, como en los encuentros de barcos en alta mar o en fondeaderos de lugares distantes, donde se intercambiarían provisiones, agua, armas, publicaciones y de diversos objetos entre los tripulantes de distintas nacionalidades. A su vez, los comerciantes marítimos y armadores compilaron informes y datos de sus capitanes, de sus socios y sus representantes comerciales en puertos de diferentes países. Si la industria ballenera puede ser caracterizada como "global" por la naturaleza de sus objetos de explotación y la organización de su actividad: persiguiendo ballenas por los distintos mares del mundo, abriendo nuevas rutas comerciales y ocupando territorios insulares, también debe ser considerada como tal en relación a la actuación de sus agentes y la circulación de información y objetos. En ese sentido, el mundo ballenero puede ser pensado como un espacio de intersección entre lo global y lo local, interconectado lugares y cosas distantes por redes de agentes, trabajadores especializados, capitales y mercancías.

La explotación de cetáceos y pinnípedos en los mares del sur compartió los patrones del comercio ultramarino y la administrada a larga distancia, mostrando parte de los engranajes de un sistema de comunicación dado por las redes establecidas por la navegación y el comercio. En las décadas de 1820 y 1830, con la multiplicación de las bases navales y los barcos de las Armadas inglesa, francesa y norteamericana 
en el Atlántico sur y el Pacífico que asisten a los balleneros de sus países, se agilizará la compilación y circulación de información sobre sus “descubrimientos" geográficos, nuevos productos y otras noticias sobre regiones distantes. En ello tuvo un papel importante el crecimiento de la prensa comercial y marítima, donde se registraba la entrada y salida de buques, sus productos, el avisaje de otros barcos, nuevas islas y localización de bajos fondos, entre otras noticias remitidas por los capitanes. A su vez, varios capitanes, pilotos u oficiales balleneros procuraron difundir el hallazgo de nuevos cotos de explotación con la intención de buscar armadores y capitalistas que financiaran alguna expedición, como se dio, por ejemplo, hacia 1819 con la rápida y amplia divulgación de noticias sobre la existencia de islas al sur del Cabo de Hornos (las Shetlands del sur) llenas de focas peleteras, elefantes marinos y ballenas (JONES, 1985).

Los capitanes balleneros tenían sus círculos de sociabilidad y espacios de reunión, donde seguramente se conversaba de los viajes y se proyectaban nuevas campañas. Esos espacios eran frecuentados por otros capitanes de ultramar, empresarios marítimos, armadores, y otros agentes de distintos rubros del comercio y la navegación marítima. Lacroix (1997, p. 172), por ejemplo, ha identificado los salones y cafés donde se reunían los capitanes balleneros en los puertos de Nantes y el Havre. Sin embargo, este tipo de espacios y su papel en la organización de empresas marítimas y en la conformación de saberes sobre los océanos son aspectos hasta ahora poco tratados en la amplia historiografía ballenera y tampoco tenidos en cuenta en la historia de las ciencias.

El éxito de las campañas balleneras dependió de varios factores. Entre ellos, fueron fundamentales la experiencia y los conocimientos acumulados por armadores y capitanes, sumado a un buen cuerpo de oficiales, arponeros y marinos (FOUCRIER; HEFFER, 2012). Pagada a la parte sobre la venta de los productos obtenidos, la tripulación estaba interesada en los resultados. La productividad también dependió de las áreas y las fechas de explotación de esos caladeros, aunque no todos los años las poblaciones de animales se presentaban o migraban de la misma forma mientras otros "bancos" mermaban por la sobreexplotación. 
La promoción, diagramación y la logística de la campaña ballenera estaba en manos del armador, frecuentemente un antiguo capitán ballenero o un miembro de una familia de empresarios balleneros o de comerciantes marítimos, propietarios de embarcaciones. Los costos financieros de comprar, equipar y asegurar un barco ballenero eran muy altos y generalmente el propietario del buque trabajaba con socios o constituía una sociedad de accionistas para afrontar los costos y riesgos del viaje. Los accionistas podían ser o no los dueños del barco, del cual en ocasiones participaban los capitanes como socios propietarios. Hubo algunos armadores de barcos balleneros que constituyeron firmas y clanes familiares que se dedicaron por muchos años a este negocio, como los Rotches y Rodman en Nueva Inglaterra, los Enderby en Inglaterra, o Winslow o Dobreé en Francia (DAVIS; GALLMAN; GLEITER, 1997; DU PASQUIER, 1982; CLAYTON; CLAYTON, 2016). En el otro extremo, hubo individuos y sociedades comerciales que solo administraron uno o dos viajes y luego dejaron la actividad ballenera.

Estos emprendedores tenían la iniciativa de las operaciones balleneras, que implicaban desde la recopilación de información precisa sobre lugares y épocas más frecuentados por los animales a explotar, los precios y mercados de sus productos, pasando por la construcción o adecuación del barco y el equipamiento, la organización y supervisión del embarque de los víveres para una larga travesía, hasta la selección del capitán y oficiales. El aprovisionamiento de comida y bebida para un viaje ballenero de dieciocho meses a tres años necesitaba una cuidadosa preparación, además de su costo. Paralelamente, el agente ballenero se ocupaba de los trámites administrativos ante las autoridades marítimas para obtener los permisos de navegación, asegurar el barco, presentar el rol de la tripulación y, en el caso de Francia o Inglaterra, gestionar la prima por el viaje, constituyendo el intermediario o referente ante los distintos organismos del Estado y los accionistas. Luego, también procuraría la venta o ubicación de los productos. Se encargaba de las operaciones logísticas antes de la partida como durante el viaje, estableciendo el itinerario del barco y/o sus escalas, y manteniendo una comunicación periódica con los capitanes en campaña y con los agentes 
comerciales ubicados en distintos puertos. Contar con una red de contactos en las escalas frecuentadas por los balleneros facilitaba que los capitanes pudieran acceder a crédito o dinero para reparaciones del barco y aprovisionamiento.

Aunque las decisiones principales estaban en manos del armador, estas podían ser discutidas o influenciadas por un capitán experimentado, especialmente si este último tenía una participación en la propiedad del barco. El proyecto de viaje incluía la duración del viaje, los lugares y épocas de pesca y las escalas donde podría reabastecerse, tomar nuevos marineros o enviar productos o noticias al armador. Aunque el itinerario se planificaba de antemano, durante el viaje se producían cambios por escases de presas, accidentes u otros sucesos desafortunados como deserciones, enfermedades o muertes, la necesidad de víveres o agua que obligaban al capitán a enfilar a un puerto. Las reparaciones del barco demandaban determinados lugares para resolverlas. A ello podían sumarse condiciones climáticas adversas, conflictos bélicos, cosarios o detenciones en puertos de otras naciones. El capitán no siempre podía esperar las instrucciones del armador antes de reaccionar a las oportunidades o desastres. Sin embargo, mantenía contacto tanto como podía, mientras el armador supervisaba de cerca el viaje a través de una administración a distancia.

Las cartas, así como periódicos, libros y diversos objetos, circularon a través de los encuentros entre barcos balleneros, aun entre los de diferentes compañías y naciones. La gran cantidad de balleneros y loberos en los mares del sur permitieron establecer un sistema de correo informal, pero de mucha eficacia. Frecuentemente, los capitanes tenían preparadas cartas para los armadores para despachar cuando se cruzaran con un barco que regresara a Europa, mientras también existía cierta especie de "buzón" en remotos lugares, como el usado por los balleneros franceses en el Estrecho de Magallanes (SINEUX, 1986).

Como se mencionaba en los relatos de viaje y en las novelas sobre balleneros, las visitas e intercambios originados al cruzarse los barcos o encontrarse en las bahías de pesca, constituyeron casi un rito ceremonial y parte de las tradiciones de la cultura marítima. Esos encuentros, 
además, del trueque de provisiones, agua, ropa o armas, facilitaron un intercambio fluido de información y consejos sobre los lugares de pesca, la abundancia y los tipos de presas y otros objetos factibles de comercialización, así como sobre la situación política de algunas zonas y los controles ejercidos sobre los balleneros. A su vez, los barcos que regresaban traían noticias sobre las embarcaciones encontradas, o cuya presencia habían escuchado a otros balleneros, junto con cartas para el armador y las familias. Además, a través de los balleneros se tendrían noticias sobre la suerte de las expediciones científicas-navales o sobre su fin, como en el caso de la expedición de Jean François Galaup, conde de La Pérouse (RENSEIGNEMENTS, 1825), o del naufragio de la de Louis Freycinet en las islas Malvinas, en este caso a través de una carta despacha en un ballenero inglés (RAPPORT, 1820).

Las cartas intercambiadas entre capitanes y sus agentes quedaban archivadas en los libros de correspondencia de los armadores, constituyendo parte de los procedimientos "en papel" de la burocracia mercantil. Los armadores podían indicarles por carta nuevos lugares de pesca o recomendarles que se dirigieran a ciertas áreas, dándole datos de longitud, latitud y época del año (FOUCRIER, 1990), según los informes recibidos de otros barcos, las noticias de los periódicos o las trasmitidas por algunos de los socios. A partir de la década de 1820, los periódicos publicados en distintos puertos constituyeron una fuente de información sobre "descubrimientos" de nuevas islas, escollos para la navegación y campos de pesca. Además, informaban sobre los precios de productos y el movimiento de barcos, incluidas noticias sobre el encuentro o naufragio de embarcaciones en distintos lugares, con lo cual los armadores podían seguir la travesía de sus barcos.

Otra fuente de información, por lo menos para los armadores franceses, fueron los diccionarios comerciales y de historia natural. Según informaba en 1819, el oficial de la Marina francesa, el barón Albin René Roussin, tras interrogar a los capitanes que encontró en Santa Catalina, uno de los puertos de descanso y aprovisionamiento preferido de los balleneros franceses en América del Sur: “Tengo entendido que la mayoría de los armadores de Francia no tienen otra opinión sobre 
esta pesca que la que extraen de los artículos de nuestros diccionarios de historia natural, donde todo lo que tiene que ver con la ballena es objeto de una amplificación casi práctica".2

Esa "amplificación casi práctica" de saberes se nutría de diferentes fuentes y la experiencia acumulada por los marinos, quienes por lo general no estaban al tanto de las últimas clasificaciones científicas, pero aprendían a distinguir desde lejos las especies empleadas en la industria, sus hábitos, migraciones y estacionalidad, lugares de reproducción o alimentación, así como varios rasgos de su anatomía (LECOMTE, 1833; BURNETT, 2007). Los diccionarios también recurrían a comentarios provistos por armadores y empresarios marítimos, sirviendo de base para nuevos aventureros. En realidad, si se tiene en cuenta la proliferación de los diccionarios de historia natural escritos por los zoólogos más importantes del siglo XIX y dirigidos tanto a los naturalistas como a los marinos, comerciantes e industriales, la transferencia de saberes "prácticos" (en diferentes direcciones) no debería sorprender: la empresa de ordenamiento y descripción científica del mundo constituyó también un proceso de inventario de recursos disponibles para el aprovechamiento humano (GARCÍA; PODGORNY, 2017).

Durante las campañas balleneras, los marinos se topaban con objetos curiosos y con cosas comunes en su oficio, las cuales, aunque aparentemente no tenían un valor económico, podía generar algún ingreso extra satisfaciendo el interés de naturalistas y coleccionistas. En sus viajes, los tripulantes y oficiales recolectaban objetos que les llamaban la atención o que obtenían del intercambio con nativos. Algunas de ellos se convirtieron en artículos comerciales bastante lucrativos, vendiéndose como curiosidades, objetos de arte o especímenes de museo, generando en algunos casos imitaciones o su producción en serie por las

2 Trad. libre de la autora: "Je conçois que la plupart des armateurs en France n'ayant d’autre opinion sur cette pêche que celle qu'ils prissent dans les articules de nos dictionnaires d'histoire naturelle, où tout ce qui a rapport à la baleine est le sujet d'une amplification presque pratique". ARCHIVES NATIONALES DE FRANCE (ANF), Paris. Extrait d'une lettre particulier écrite de Rio-Janeiro le 24 juin 1819, par Mr. Roussin, commandant de la Corvette La Bayadère, 1819. Fond Marine Microfilms (MMAR), Sous-séries CC5, Microfilm 593. 
comunidades locales. Recordemos, por ejemplo, las llamadas "cabezas maoríes", cabezas humanas disecadas y decoradas con tatuajes preparadas por indígenas de Nueva Zelanda y que los balleneros llevaron a Europa y Estados Unidos en las décadas de 1820 y 1830. Paralelamente en Santa Catalina, las tripulaciones de los barcos balleneros franceses capturaron numerosas aves para comercializar en los puertos franceses (LACROIX, 1997). En otros casos domesticaron algún animal de la fauna local como mascota o para exhibir en el siguiente puerto donde recalaran. Muchos marinos participarían en las exhibiciones itinerantes de animales. Otros objetos curiosos recolectados en una localidad servirían para regalar a las autoridades portuarias de otra zona, para pagar algún favor o agasajar a alguna novia. Algunas muestras llegarían a los museos y a manos de los naturalistas. Por ejemplo, el capitán lobero, James Weddell, al regresar de los mares antárticos depositó algunas muestras de rocas y animales en el Museo de la Universidad de Edimburgo. Entre ello se encontró una nueva especie de foca que fue bautizada en su honor: Leptonychotes weddellii. Weddell, desde 1819, navegó por los mares australes, reuniendo informes y observaciones de las zonas visitadas y publicando una memoria de sus viajes en 1825 (GARCÍA; PODGORNY, 2017).

Información especial sobre las áreas de pesca era comunicada por capitanes y oficiales balleneros y coleccionada por los armadores. Por ejemplo, como mencionan Davis; Gallman; Gleiter (1997), la compañía norteamericana Aiken y Swift acumuló un conjunto de notas sobre los "bancos" de pesca que llegaron a conformar cuatro volúmenes. Las notas solían describir sucintamente el avistaje y las capturas de ballenas, con sus coordenadas geográficas y fecha y productos obtenidos. Estos ítems a registrar conformaban parte de las instrucciones que daban los armadores a sus oficiales y se anotaban en los diarios de navegación. Así, por ejemplo, en 1821, uno de los principales empresarios balleneros del Havre, el nantokois Jeremiah Winslow, recomendaba al segundo capitán de uno de sus navíos destinado a las costas de Brasil, que pusiera mucho empeño en la confección del diario de viaje, indicando los principales puntos a completar: "Debe tener mucho cuidado al redactar su Diario, 
y anotar con exactitud todos los objetos que se relacionan con la pesca, la hora, latitud y longitud en la que se captura cada ballena, la cantidad de aceite que produce cada una".

Instrucciones similares se dieron para el viaje del barco ballenero Triton, de Nantes, en 1822. Este barco fue comandado por el capitán norteamericano John Upham y como segundo capitán se embarcó a un ciudadano francés con diploma de capitán de ultramar. Este último recibió del secretario de la casa Dobrée la orden de llevar en francés un diario exacto de las operaciones, debiendo registrar fechas, posiciones geográficas, cantidades de ballenas capturadas, horas de fundición, útiles perdidos, entre otros detalles (LACROIX, 1997, p. 224).

Llevar un registro diario a bordo era una obligación del capitán y/o algunos oficiales, demandada por los armadores y las compañías aseguradoras. En Francia, además, servía para cobrar la subvención del gobierno a los viajes balleneros, cuyo monto aumentaba si se demostraba que se había operado más allá de los cabos de Hornos y Buena Esperanza o de los $60^{\circ}$ de Latitud sur. Al retornar de los viajes, el armador o el capitán debía presentar por escrito a las autoridades portuarias una "declaración de retorno", un pequeño resumen del viaje (SINEUX, 1986). Algunos capitanes adjuntaban su diario o cuaderno de bitácora como respaldo de ese relato. ${ }^{4}$ Los funcionarios de los puertos franceses

3 Trad. libre de la autora: "Il faut apporter beaucoup de soin à la rédaction de votre Journal, et noter avec exactitude tous les objets que ont rapport à la pêche, lépoque, la latitude et la longitude auxquelles chaque baleine est prise, la quantite d'huile que chacune d'elles produit". ANF, Paris. Copie des instructions à Monsieur Maupas, Second Capitaine du navire baleinier George et Albert, données par J. Winslow, 1821. MMAR, Sous-séries CC5, Microfilm 593. Ese barco fue comandado por el norteamericano Seth Fisher y realizó un viaje ballenero al "banco" de Brasil y Santa Catalina, partiendo el 23 de octubre de 1821 y retornando el 23 de febrero de 1823.

4 Por ejemplo, en 1822, el capitán norteamericano Latham Paddock, comandante del barco Bourbon despachado por Winslow, presentó ante las autoridades portuarias del Havre su declaración de retorno y su diario de navegación como respaldo. El oficial de la Marina en ese puerto, después de interrogar a los hombres de la tripulación y haber comparado sus declaraciones con las del capitán y su diario, certificó que había cumplido con las condiciones estipuladas en la ordenanza real del 14 de febrero de 1819. ANF, Paris. Déclaration de retour du capitaine du navire baleinier Le Bourbon, 1822. MMAR, Sous-séries CC5, Microfilm 593. 
podían recurrir a esos documentos para corroborar las declaraciones de retorno, junto con los interrogatorios al capitán, oficiales y otros miembros de la tripulación, a fin de establecer si se habían cumplido las condiciones para el pago de la prima. Por otra parte, el diario de navegación era parte de los papeles que las autoridades marítimas de distintos puertos podían exigir examinar, al igual que los barcos de guerra con los que se cruzasen. No disponer a bordo de este tipo de documento, implicaba que la embarcación se dedicaba a alguna actividad ilegal, como contrabando y piratería. Como los diarios de navegación debían presentarse ante autoridades portuarias, navales, agentes consulares y los que patrocinaban el viaje, muchos de ellos contaron con varias copias o partes de ellas que circularon por distintas manos y archivos. Además, algunos capitanes y oficinales guardaron sus versiones originales o sus copias como una documentación que probaba sus antecedentes náuticos.

Los diarios de navegación (cuadernos de bitácora) eran una herramienta técnica importante de los navegantes y formaba parte de la cultura marítima desde por lo menos el siglo XVII, prescritos en ordenanzas navales y vinculados con la práctica de la "estima" (SCHOTTE, 2013; CHALINE, 2016). Esto implicaba una anotación, día a día, de la latitud y longitud estimadas y/o observadas, los rumbos seguidos y las distancias recorridas para deducir la posición veinticuatro horas después, aun cuando no se pudieran realizar mediciones astronómicas para calcular la ubicación geográfica del barco. En ese sentido, podría decirse que el registro de estos datos conformaba parte del "instrumental de navegación”, en tanto permitían estimar el itinerario del barco. Como sugiere Chaline (2016) no debe olvidarse que la navegación astronómica y la determinación de la longitud, sobre todo a fines del siglo XVIII, demandaba competencias científicas cada vez más exigentes manejabas por una elite e instrumentos costosos que hacía difícil su aplicación fuera de las expediciones científicas y los barcos de la armada o de las compañías de Indias. La insistencia de la historiografía sobre los nuevos instrumentos de navegación, señala este autor, es inversamente proporcional a su frecuencia (CHALINE, 2016, p. 63). Muchos navegantes 
continuaron recurriendo a métodos tradicionales e incorporando procedimientos simplificados. ${ }^{5}$ La historia de la ciencia ha dejado de lado la supervivencia de ese otro tipo de prácticas de navegación que coexistieron con la expansión del instrumental para la navegación astronómica, los cronómetros marinos y la determinación de la longitud. En ese sentido, queda para futuras investigaciones inventariar los instrumentos y prácticas de navegación de los barcos balleneros en los mares del sur. En la década de 1820, balleneros y loberos portaban algún cronómetro y practican algún tipo de medición de la longitud, aunque no todos con mucha precisión. El lobero inglés James Weddell (1825), por ejemplo, menciona entre sus instrumentos de "empleo corriente" el uso de 3 cronómetros (uno de 8 días, otro de dos días y el tercero de 24 horas) en su navegación hacia los mares antárticos. Tal vez los antecedentes de este lobero como oficial en barcos mercantes y de la armada inglesa y su interés por las ciencias hacen que no pueda generalizarse su experiencia al promedio de los loberos y balleneros de la época, aunque algunos reconocían que:

5 El problema de la determinación del tiempo en los largos viajes seguía persistiendo al terminar el siglo XVIII, aun cuando hacía varias décadas que se había inventado y perfeccionado el cronómetro marino. No obstante, su alto costo lo hacía prohibitivo para gran parte de los navegantes. Por otra parte, en los largos viajes era difícil chequear el error del cronómetro, para lo cual se necesitaba contar con varios de ellos y de diferentes modelos y fabricantes, y, además, cotejar con el método de la "distancia lunar", basado en la observación de la distancia de la luna al sol y algunas estrellas fijas. Muchos los barcos navegaban con una combinación de "cálculo muerto" (dead reckoning) y navegación por paralelo (un sistema de navegar al norte o sur por la latitud de un destino y al este u oeste de ese punto). En las sucesivas ediciones de manuales para la "navegación práctica", se fueron compilando tablas de logaritmos y corrigiendo errores de cálculo para facilitar la determinación de la latitud y longitud, como en el texto de John Hamilton Moore: MOORE, John Hamilton. The Practical Navigator and Seaman's New Daily Assistant. London: W \& J Richardson, 1772. Uno de los más populares, y la versión norteamericana de Nathaniel Bowditch: BOWDITCH, Nathaniel. The New American Practical Navigator. Newburyport: Edmund M. Blunt, 1802. Pequeños errores por unos minutos en las tablas de logaritmos podían causar grandes tragedias. En el manual de Bowditch, además de corregirse algunos de esos errores, se incluyó un procedimiento simplificado para que los marinos tomaran la distancia lunar y calcularan la posición del barco. 
Los balleneros están mucho más avanzados en matemáticas y navegación práctica que otros navegantes, ya que, en sus largos viajes de ida y vuelta, los oficiales más inteligentes ayudan a los más jóvenes en sus estudios matemáticos y náuticos; y así instruidos, todos vuelven a casa mejorados en sus ramas, siendo la distinción en ellas el camino directo hacia el ascenso. ${ }^{6}$

Los barcos loberos y balleneros constituyeron una escuela náutica (y en algunos casos también de primeras letras) para los jóvenes novicios y los niños de cámara. Los sectores balleneros enfatizarían que la navegación y su oficio se aprendía en el mar. En el caso de los balleneros nantokois: "en sus largos viajes, los jóvenes estudian matemáticas, navegación, geografía y la historia de los Mares del Sur, en relación con su peligrosa profesión. Hay muchos conocimientos reales sobre estos puntos, comunicados por los marineros mayores a los más jóvenes"?

En los inicios del siglo XIX, los manuales para la navegación "práctica" brindaban instrucciones y tablas de logaritmos para la determinación de la latitud y asegurar la longitud por la observación lunar y

6 Trad. libre de la autora: "the whalemen are much advanced in mathematics and practical navigation beyond other navigators: for, on their long voyages out and home, the most intelligent officers assist the younger in their mathematical and nautical studies; and thus schooled, all come home improved in their branches, distinction in them being the direct road to preferment". Comentario de Jeremiah N. Reynolds en un informe sobre los descubrimientos geográficos de los balleneros presentado al secretario de la Armada norteamericana el 24 de septiembre de 1828. Este documento fue luego reproducido en INFORMATION Collected by the Navy Department Relating to Islands, Reefs, Shoals, Etc., in the Pacific Ocean and South Seas, and Showing the Expediency of an Exploring Expedition in that Ocean and those Seas by the Navy, 29 Jan. 1835. In: American State Papers. Documents, legislative and executive of the Congress of the United States from the Second session of the Twenty-First to the First session of the Twenty-Fourth Congress, commencing March 1, 1831, and ending June 15, 1836. Naval Affairs, v. IV. Washington: Gale’s \& Seaton, 1861, p. 688-700. Disponible en: < http://whalesite.org/anthology/usexex573.htm >. Acceso en: 3 ago. 2021.

7 Trad. libre de la autora: "in their long voyages, the Young men study mathematics, navigation, geography and the history of the South Seas, as connected with their hazardous profession. There is much real knowledge on these points, communicated from the older to younger sailors." THE WHALE Fishery. Nantucket Inquiry, Nantucket, 14 Feb. 1829, p. 1. 
otros métodos; la manera de encontrar y conocer los planetas y estrellas fijas en ambos hemisferios; el arte de mapear costas y fondeaderos, así como la forma de llevar un diario de navegación, entre otros temas. Por entonces, los diarios de los barcos balleneros tienen una forma bastante estandarizada. En esos cuadernos, se consignaban escuetamente los acontecimientos diarios de la vida a bordo y se anotaba sistemáticamente por cada día náutico una serie de datos técnicos: fecha y hora, puntos geográficos (latitud y longitud calculada o estimada), rumbos tomados, fuerza y dirección de los vientos, estado del tiempo y del mar, velamen, profundidades (sondajes) cerca de la costa, y declinación de la aguja. A veces se acompañaban de tablas o listados que resumían los datos meteorológicos, la ubicación de islas y rocas recientemente descubiertas y los resultados de la pesca. Muchos contenían ilustraciones de ballenas y otros animales, barcos, vistas de costas o planos de islas y fondeaderos (COMERLATO, 2019).

Ciertos capitanes llevaron diarios con descripciones más extensas de los lugares visitados, registrando instrucciones para la entrada a puerto o pasar determinado paraje e incluyendo esquemas de los perfiles de las costas. Otros escribirían relatos de sus viajes para matar el tedio de la navegación o para entretener a sus familias y amigos. Algunos capitanes balleneros y loberos publicaron las memorias de sus viajes, un género literario que se popularizó en la década de 1820. Otros miembros de la tripulación, por lo menos los sectores letrados, también escribieron sobre sus aventuras y lugares visitados.

Los médicos embarcados en los balleneros franceses e ingleses publicaron relatos de sus viajes y observaciones científicas (FORSTER, 1988; SINEUX, 1986). Entre esto último, por ejemplo, se puede mencionar la historia natural del cachalote (BEALE, 1839) o la identificación científica de los organismos que, según los balleneros, formaban parte del alimento de las ballenas en el Atlántico sur y su abundancia marcaba los llamados "bancos” de ballenas (ROUSSEL DE VAUZÈME, 1834). A diferencia de las campañas balleneras norteamericanas, las reglamentaciones navales tanto de Gran Bretaña como de Francia prescribían la obligación de llevar un cirujano en los navíos de comercio, incluidos 
los balleneros. En el caso de Francia, las ordenanzas de 1819 exigían la presencia de un cirujano en viajes largos de pesca con más de 20 tripulantes, el cual debía gozar del estatus de un oficial a bordo y compartir su mesa y alimentación. Sus obligaciones, además de atender a la salud de la tripulación, incluían ocuparse de la conservación de los víveres y de la caja de los medicamentos, así como llevar un registro diario de los acontecimientos, enfermedades y tratamientos que se dieran durante el viaje. $\mathrm{Al}$ igual que para los capitanes, confeccionar un diario de a bordo era inherente a la profesión de la medicina naval y debía ser presentado ante las autoridades sanitarias portuarias para su verificación y aprobación (LECOMTE, 1833, p. 46).

\section{LA RECOPILACIÓN Y CIRCULACIÓN DE LA INFORMACIÓN OCEÁNICA}

Tal como se mencionó anteriormente, la actividad ballenera generó diversos registros escritos: bitácoras, diarios, cartas, tablas de pesca, declaraciones de retorno, interrogatorios, rectificación de las posiciones geográficas o noticias sobre hallazgo de islas no marcadas en las cartas náuticas y de peligros para la navegación, entre otros documentos. La información sobre nuevas islas, arrecifes o rocas detectados por los balleneros fueron incorporados a los archivos de las expediciones científicas-navales, y otros fueron publicados en los relatos de viajes, en periódicos marítimos y comerciales de la época o en anales académicos. Parte de esos materiales y los diarios de navegación fueron usados o consultados por sectores científicos y navales para el estudio de distintos fenómenos. Por ejemplo, el periódico francés L’Echo du Monde Savant, señalaba que los diarios de navegación y la experiencia del capitán ballenero francés, Simon Coste, habían ayudado a esclarecer los efectos de los terremotos de 1835 y 1837 en el litoral chileno: "El capitán ballenero Coste, actualmente al mando del Ocean, lleva muchos años frecuentando la costa chilena; leyendo sus diarios hemos podido 
también reunir datos que no dejarán lugar a dudas sobre los levantamientos que siguen a los terremotos".

Esos datos y otros registros sobre los temblores de tierra, seguidos de procesos de levantamientos y hundimientos, en Chile fueron reunidos en Valparaíso por el ingeniero hidrógrafo de la corveta francesa l'Astrolabe, durante la segunda expedición científica comandada por Jules Dumont d'Urville, y enviados a la Academia de Ciencias de Paris. En las décadas de 1820 y 1830, con la multiplicación de las bases navales, las expediciones científicas y los barcos de guerra ingleses, franceses y norteamericanos en el Atlántico sur y el Pacífico se agilizará la compilación y circulación en múltiples direcciones de los "descubrimientos" geográficos y otras observaciones de los balleneros, loberos o los prácticos de diferentes puertos, algunos de los cuales además fueron contratados para los levantamientos hidrográficos de costas difíciles de mapear (GARCÍA, 2010; 2013; GARCÍA; PODGORNY, 2014). Esas noticias no se guardaron como secreto, por el contrario, se trasmitieron entre comandantes, oficiales y embajadores de distintas nacionalidades. Así, por ejemplo, en 1825 el teniente francés Louis M. Barral envió desde Chorrillos (cerca de Lima, Perú) al Ministerio de Marina de Francia información sobre arrecifes de la costa peruana y una lista de "las posiciones de diferentes islas, arrecifes y rocas descubiertas por los barcos balleneros en el Mar del Sur". ${ }^{9}$ Esta recopilación se la había pasado el comandante de la escuadra norteamericana en Valparaíso, quien a su vez la había obtenido de un capitán ballenero de Nantucket.

La lista consignaba la latitud y longitud de más de 30 islas y arrecife y rocas denominadas con los apellidos de balleneros y loberos, armadores o los nombres de sus barcos. Barral consideraba que no todos

8 Trad. libre de la autora: "Le capitaine baleinier Coste, commandant aujourd'hui l'Océan, depuis nombre d’années fréquente les parages de la côte du Chili; en parcourant ses journaux nous avons pu y recueillir aussi des données qui ne laisseront aucun doute sur les soulèvements à la suite des tremblements de terre". PHYSIQUE du Globe. Tremblement de terre du Chile. L'Echo du Monde Savant, 14 nov. 1838. T. 5, n. 45, p. 329.

9 ANF, Paris. Frégate Marie-Thérèse: travaux hydrographiques du lieutenant de vaisseau Barral, 1825. Fond Marine (MAR), Sous-série Marine 5JJ, dossier 217. 
eran nuevos descubrimientos sino islas marcadas con otros nombres y posiciones en las cartas náuticas. Asimismo, desconfiaba de los datos de longitud: "Yo no sé cómo las longitudes de estos diferentes puntos habían sido obtenidas". ${ }^{10}$

A pesar de esa desconfianza, la lista de "descubrimientos" fue incorporada al Depósito de Cartas de la Marina francesa y examinada por expertos de esa dependencia que consideraron que se trataba de información muy útil a la navegación. La lista fue luego copiada y entregada al comandante Dumont d'Urville para la corrección de las cartas del gran océano durante su expedición en la corveta Astrolabe. ${ }^{11}$ En el Depósito de Cartas también se coleccionaron otras noticias sobre islas y rocas descubiertas por balleneros norteamericanos dadas a conocer en el periódico Nantucket Inquirier, reunidas y traducidas por los diplomáticos franceses de Nueva York y Washington. ${ }^{12}$ Otras compilaciones de los descubrimientos en el Océano Pacífico fueron realizadas por las expediciones navales francesas interrogando a los prácticos locales y a los capitanes balleneros. En 1836, durante la expedición de La Bonite, en la rada de Honolulu se reunió una lista de islas y peligros descubiertos por los balleneros con las posiciones geográficas asignadas por ellos. A la longitud indicada según el meridiano de Greenwich, los oficiales franceses le agregaron la del meridiano de Paris. ${ }^{13}$ Aunque los saberes y prácticas náuticas de los balleneros fueron cuestionados por oficiales de la marina francesa, especialmente por aquellos que promovían la profesionalización del trabajo hidrográfico y una formación académica

10 Trad. libre de la autora: "Je n’ai pas savoir comment les longitudes de ces différents points avaient été obtenus". ANF, Paris. Frégate Marie-Thérèse: travaux hydrographiques du lieutenant de vaisseau Barral, 1825. MAR, Sous-série Marine 5JJ, dossier 217.

11 ANF, Paris. Positions des iles, récifs et rochers découverts dans le grand océan, 1825. MAR, Sous-série Marine 5JJ, dossier 217.

12 ANF, Paris. Campagnes et travaux divers, 1817-1871. MAR, Sous-série Marine 5JJ, dossier 365.

13 ANF, Paris. Table des îles et dangers découverts par des baleiniers, 1837. MAR, Sous-série Marine 5JJ, dossier 159. 
(licencia de navegación oceánica) ${ }^{14}$ de los capitanes balleneros, al mismo tiempo los diarios y registros de los balleneros fueron solicitados para la corrección de cartas, chequear la ubicación de islas u otros accidentes geográficos, para estudios de meteorología y aún hasta para analizar los efectos de los terremotos en la costa chilena. Por otra parte, la circulación de esas noticias y datos muestra que los "descubrimientos" asociados a determinada expedición naval o capitán en realidad deben pensarse como conocimientos acumulados colectivamente y que condensan capas de distintos tipos de saberes y prácticas.

La compilación de información provista por los balleneros se repetía en otros lugares. En 1828, un antiguo periodista y delegado del Departamento de Marina de Estados Unidos, Jeremiah Reynolds, visitó los puertos de Nueva Inglaterra para interrogar a los capitanes y propietarios de barcos despachados a los mares del sur:

Me dirigí sin demora a New London, Stonington, Newport, New Bedford, Edgartown, Nantucket y otros lugares donde se podía encontrar información sobre el Océano Pacífico y los mares del Sur. Los capitanes balleneros estaban dispuestos a comunicar los conocimientos que habían atesorado o registrado en sus numerosos viajes. Los propietarios de los barcos balleneros estaban igualmente dispuestos a hacer todo lo posible para ayudarme en el objetivo de mi visita. En estos lugares, los navegantes conocen ciertamente mejor esos mares de lo que puede conocer cualquier otro pueblo de este u otro país. La información se había reunido, en cierta medida, en bruto, sin orden ni mucha disposición; y tuve que recorrer todo el terreno, y examinar en Nantucket a cada uno de los navegantes de esos mares que se podían encontrar en su casa, con sus cuadernos de bitácora, diarios y cartas de navegación. Los primeros objetos de mi investigación fueron la navegación, la geografía y la topografía presentes en toda

14 Trad. libre de la autora: "brevet de long-cours". 
la variedad de los mares, desde el Pacífico hasta los océanos Índico y chino; también, la extensión y la naturaleza de nuestro comercio y pesca en estos mares. ${ }^{15}$

Reynolds calculaba que en esa época al menos unos 200 barcos norteamericanos estaban empleados en la actividad ballenera, incluyendo los del comercio lobero. Además de las conversaciones y la consulta de los diarios y cartas náuticas de los balleneros, envió cartas e instrucciones para obtener información de otros capitanes. El comisionado de Washington reconocería la inexactitud de algunos de los datos y la falta de sistematización de la información reunida entre los balleneros, siendo necesario confrontar las fuentes de información:

La información que he recogido, si no es perfectamente exacta, es sin duda la más exacta que se puede encontrar. Se ha extraído de fuentes puramente originales; nada se ha recibido de segunda mano. He examinado los cuadernos de bitácora, los diarios, los mapas y las cartas de los propios

15 Trad. libre de la autora: "I repaired without delay to New London, Stonington, Newport, New Bedford, Edgartown, Nantucket, and other places where information might be found of the Pacific Ocean and South seas. The whaling captains were ready to communicate such knowledge as they had treasured up or recorded in their numerous voyages. The owners of the whale ships were equally anxious to do all in their power to assist me in the object of my visit to them. In these places the navigators are certainly better acquainted with those seas than any other people in this or any other country can be. The information had, in some measure, been gathered in gross, but without order or much arrangement; and I had to go over the whole ground, and examine at Nantucket every individual navigator of those seas who could be found at home, with their log books, and journals and charts. The first objects of my inquiry were the navigation, geography, and topography presented by the whole range of the seas, from the Pacific to the Indian and Chinese oceans; also, the extent and nature of our commerce and fisheries in these seas". INFORMATION Collected by the Navy Department Relating to Islands, Reefs, Shoals, Etc., in the Pacific Ocean and South Seas, and Showing the Expediency of an Exploring Expedition in that Ocean and those Seas by the Navy, 29 Jan. 1835. In: American State Papers. Documents, legislative and executive of the Congress of the United States from the Second session of the Twenty-First to the First session of the Twenty-Fourth Congress, commencing March 1, 1831, and ending June 15, 1836. Naval Affairs, v. IV. Washington: Gale’s \& Seaton, 1861, p. 688. 
navegantes, y en la mayoría de los casos los he interrogado personalmente (...). Cuando los individuos eran igualmente buenos en cuanto a inteligencia, y sus declaraciones diferían de alguna manera, he dado ambos relatos; pero si había una diferencia decisiva en la inteligencia de la autoridad, he adoptado el que tenía la estimación más general. ${ }^{16}$

Más allá de los problemas de la divergencia e imprecisión de los registros, el delegado de la Marina norteamericana consignó una lista de más de 280 islas, arrecifes y rocas descubiertas por balleneros y no consignadas en las cartas náuticas inglesas usadas en esa época. Como ha analizado Felix Lüttge (2019), abstrayendo la información de forma concisa, confrontándola con otras fuentes de datos y enumerándola en una lista, Reynolds convirtió las noticias de los balleneros en hechos y las islas en objetos epistémicos. Aunque esa información recopilada presentaba cierta inexactitud, resultó útil para estimular una primera exploración científica financiada por el gobierno norteamericano en 1838 (KUGLER, 1971). También antes de una segunda expedición oficial en 1852, el comandante de la misma visitó New Bedford para discutir con los capitanes balleneros sus conocimientos sobre las regiones a explorar (KUGLER, 1971). Por esa época, el teniente Matthew Fontaine Maury, encargado del Depósito de Cartas e Instrumentos de la Armada norteamericana, utilizó los datos consignados en los diarios

16 Trad. libre de la autora: "The information I have collected, if not perfectly accurate, is certainly the most so that can be found. It has been drawn from purely original sources; nothing has been received at second hand. I have examined the log books, journals, maps, and charts of the navigators themselves, and in most cases have questioned them personally (...). When the individuals were equally good in point of intelligence, and their statements in any way differed, I have given both accounts; but if there was a decided difference in the intelligence of the authority, I have adopted that which was held in the most general estimation". INFORMATION Collected by the Navy Department Relating to Islands, Reefs, Shoals, Etc., in the Pacific Ocean and South Seas, and Showing the Expediency of an Exploring Expedition in that Ocean and those Seas by the Navy, 29 Jan. 1835. In: American State Papers. Documents, legislative and executive of the Congress of the United States from the Second session of the Twenty-First to the First session of the Twenty-Fourth Congress, commencing March 1, 1831, and ending June 15, 1836. Naval Affairs, v. IV. Washington: Gale's \& Seaton, 1861, p. 689. 
de navegación disponibles entre 1802 y 1850, para elaborar cartas de vientos y corrientes que facilitaran la "navegación práctica" (PINSEL, 1981). Asimismo, recurrió a un cuerpo de observadores, reclutados del servicio mercante pero especialmente entre capitanes balleneros que podían proveer datos de regiones distantes y diferentes de las atravesadas por las rutas comerciales. Poco después, Maury publicó una carta con la distribución mundial y estacional de los dos principales tipos de cetáceos explotados y diferenciados en los registros balleneros (la ballena franca y el cachalote) (ROUDER, 1980). Esos mapas se ofrecieron en venta a los balleneros, pero también fueron usados por los naturalistas. Ese oficial y sus asistentes (uno de ellos un capitán ballenero retirado) procesaron la información contenida en cientos de diarios de navegación y la información provista por los balleneros, transformándola en marcas en una carta náutica. De esta forma, la distribución espacial y estacional de las ballenas en los océanos adquirió una "consistencia óptica" (LATOUR, 1990), naturalizándose su existencia geográfica a partir de su caza.

Siguiendo el ejemplo de Maury, en la década de 1920 Charles Haskins Townsend y su ayudante Arthur C. Watson, de la Sociedad Zoológica de Nueva York, recogieron datos de los cuadernos de balleneros entre 1780 y 1920, para confeccionar mapas e ilustrar la distribución global de las ballenas (TOWNSEND, 1935). Una iniciativa retomada en el siglo XXI en el marco de los censos de la vida marina (SMITH et al., 2012). Anteriormente en las décadas de 1970 y 1980, en el seno de la Comisión Internacional Ballenera se discutió la importancia analítica de los registros históricos de los balleneros (TILLMAN; DONOVAN, 1983). Otras investigaciones apelan también a esos documentos para la estimación histórica y evolución de las poblaciones de cetáceos y pinnípedos, así como para estudios de meteorología, los contactos interétnicos, la historia ambiental de las islas oceánicas, entre otros tópicos. Los diarios de los balleneros del siglo XIX continuaron consultándose y compilándose en el siglo XX y aún a inicios del siglo XXI, constituyéndose en objetos de colección, pero también en herramientas epistémicas para estudiar los océanos desde una escala global. 


\section{RECONOCIMIENTOS}

Parte de este trabajo se basó en materiales consultados en los Archivos Nacionales de Francia y Gran Bretaña y las bibliotecas de los museos marítimos de Paris y Londres. La visita a dichos archivos fue posible gracias a los recursos de los proyectos de investigación: PIP 0153, PICT 2015-3534, Eco-Sud A15H02 y del Programa "Malvinas en la Universidad" de Secretaría de Asuntos Relativos a las Islas Malvinas del Ministerio de Relaciones Exteriores y Culto de la Nación y el Ministerio de Educación de la Argentina. Asimismo, este trabajo forma parte del Proyecto RISE SciCoMove (Scientific Collections on the Move), dirigido por Nathalie Richard e Irina Podgorny, financiado por la Unión Europea a través de su programa Horizonte 2020 para la investigación e innovación científicas y el subsidio Marie Sklodowska-Curie N 1011007579. Por último, agradezco los sugerentes comentarios y las indicaciones bibliográficas de los evaluadores anónimos de este artículo.

\section{REFERENCIAS BIBLIOGRÁFICAS}

BEALE, Thomas. The Natural History of Sperm Whale. Londres: John Van Voorst, 1839.

BOWDITCH, Nathaniel. The New American Practical Navigator. Newburyport: Edmund M. Blunt, 1802.

BURNETT, Graham D. Trying Leviathan: the Nineteenth-Century New Court Case that put the Whale on Trial and Challenged the Order of Nature. Princeton: Princeton University Press, 2007.

CASTELLUCCI JUNIOR, Wellington. Caçadores de baleia: armações, arpoadores, atravessadores e outros sujeitos envolvidos nos negócios do cetáceo no Brasil. São Paulo: Annablume, 2010.

CASTELLUCCI JUNIOR, Wellington. Histórias conectadas por mares revoltos: uma história da caça de baleias nos Estados Unidos e no Brasil (1750-1850). Revista de História Comparada, Rio de Janeiro, v. 9, n. 1, p. $88-118,2015$. 
CATALOGUE of Nantucket Whalers and their Voyages from 1815-1870. Nantucket: Hussey \& Robinson, 1876.

CHALINE, Oliver. La Mer et la France. Quand les Bourbons voulaient dominer les océans. Paris: Flammarion, 2016.

CLAYTON, Jane. Nantucket Whalers in Milford Haven, Wales. Historical Nantucket, v. 56, n. 1, p. 4-7, 2007.

CLAYTON, Jane M.; CLAYTON, Charles A. Shipowners investing in the South Sea Whale Fishery from Britain: 1775-1815. Hassobury: Jane M. Clayton, 2016.

COMERLATO, Fabiana. O desenho de baleias nos diários de bordo de navios baleeiros norte americanos do século XIX. In: COMERLATO, Fabiana; QUIROZ, Daniel (Org.). Baleias e baleeiros: patrimônio cultural e conservação ambiental. Pelotas: BasiBooks, 2019. p. 10-26.

COMERLATO, Fabiana; QUIROZ, Daniel (Org.). Baleias e baleeiros: patrimônio cultural e conservação ambiental. Pelotas: BasiBooks, 2019.

DAVIS, Lance E. ; GALLMAN, Robert E. ; GLEITER, Karin. In Pursuit of Leviathan: Technology, Institutions, Productivity, and Profits in American Whaling, 1816-1906. Chicago: University of Chicago Press, 1997.

DICKINSON, Anthony. Seal Fisheries of the Falkland Islands and Dependencies: A Historical Overview. St John's: International Maritime Economic History Association, 2007.

DU PASQUIER, Jean Thierry. Les Baleiniers français au XIX $X^{\mathrm{e}}$ siècle (1814-1868). Grenoble: Terre et Mer; 4 Seigneurs, 1982.

DU PASQUIER, Jean Thierry. Les Baleiniers français de Louis XVI à Napoléon. Paris: Henri Veyrier, 1990.

EDMUNDSON, William; HART, Ian. A História da caça de baleias no Brasil: de peixe real a iguaria japonesa. Barueri: DISAL, 2014.

ELLIS, Miriam. A Baleia no Brasil Colonial. São Paulo: Edusp, 1968.

FOUCRIER, Annick. Baleiniers français en Californie (1825-1848). Revue d'histoire moderne et contemporaine, v. 37, n. 2, p. 239-252, 1990.

FOUCRIER, Annik; HEFFER, Jean. La Productivité de la pêche à la baleine française, 1817-1868. Histoire \& Mesure, v. 27, n. 2, p. 49-77, 2012.

FORSTER, Honore. British Whaling Surgeons in the South Seas, 1823-1843. Mariner's Mirror, v. 74, n. 4, p. 401-415, 1988. 
GARCIA, Susana V. La Logística de los levantamientos hidrográficos en el Río de la Plata y Patagonia en tiempos del HMS Beagle. Anuario IEHS, Tandil, v. 25, p. 301-324, 2010.

GARCIA, Susana V. Los Prácticos del Río Negro y la circulación de información (1820-1840). In: WEISSEL, Marcelo (Comp.). Temas de patrimonio cultural 30: Argentina de Puertos. Buenos Aires: Comisión para la Preservación del Patrimonio Histórico-cultural, 2013. p. 225-244.

GARCIA, Susana V.; PODGORNY, Irina. Los Pilotos del Río Negro y las escorias de la Patagonia. In: ACHIM, Miruna; PODGORNY, Irina (Ed.) Museo al detalle. Colecciones, antigüedades e historia natural, 1790-1870. Rosario: Prohistoria, 2014. p. 127-156.

GARCIA, Susana V.; PODGORNY, Irina. L'Exploitation de la faune marine dans l'Atlantique Sud: quelques idées pour une histoire globale de la zoologie et du commerce au XIX ${ }^{\mathrm{e}}$ siècle. In: ROJAS, Daniel (Ed.). Amérique Latine globale. Histoire connectée, globale et internationale. Paris: Harmattan, 2017. p. 17- 47.

JACKSON, Gordon. The British Whaling Trade. Londres: Adam \& Charles Black, 1978.

JENKINS, James T. A History of the Whale Fisheries: from the Basque Fisheries of the Tenth Century to the Hunting of the Finner Whale at the Present Date. Londres: H. F. and G. Witherby, 1921.

JONES, A. G. E. Age British Sealing on New South Shetland 1819-1826. The Great Circle, v. 7, n. 1, p. 9-22, 1985.

KUGLER, Richard C. The Penetration of the Pacific by American Whalemen in the $1^{\text {th }}$ century. Maritime Monographs and Reports, n. 2, p. 1-32, 1971. LATOUR, Bruno. Drawing Things Together. In: LYNCH, Michael; \WOOLGAR, Steve (Org.). Representation in Scientific Practice. Cambridge \& London: MIT Press, 1990. p. 19-68.

LACROIX, Louis. Les Derniers baleiniers français. Rennes : Ouest-France, 1997.

LECOMTE, Jules. Pratique de la pêche à la baleine dans les mers du Sud. Paris: Lecointe et Pougin, 1833. 
LÜTTGE, Felix. Whaling Intelligence: News, Facts and US-American Exploration in the Pacific. British Journal of History of Science, v. 52, n. 3 , p. 425-445, 2019.

MOORE, John Hamilton. The Practical Navigator and Seaman's New Daily Assistant. London: W \& J Richardson, 1772.

PINSEL, Marc I. The Wind and Current Chart Series Produced by Matthew Fontaine Maury. Navigation: Journal of the Institute of Navigation, v. 28, n. 2, p. 123-137, 1981.

PODGORNY, Irina. Las Instrucciones y las cosas. Revista Hispánica Moderna, v. 71, n. 1, p. 23-38, 2018.

PODGORNY, Irina. Bureaucracy, Instructions, and Paperwork. The Gathering of Data about the Three Kingdoms of Nature in the Americas, 1770-1815. Nuevo Mundo Mundos Nuevos, 2019. Disponible en: $<$ https://journals.openedition.org/nuevomundo/75454>. Acesso en: 28 jul. 2021.

QUIROZ, Daniel. Operaciones balleneras tradicionales en las costas sudamericanas. Estudios Atacameños, n. 65, p. 47-64, 2020.

QUIROZ, Daniel; TOLEDO, Patricio (Ed.). Balleneros del Sur. Antropología e historia de la industria ballenera en las costas sudamericanas. Santiago de Chile: Andros, 2014.

RAPPORT adressé à Son Exc. Le Ministre de la marine et des colonies, par M. Louis de Freycinet, Capitaine de Frégate. Annales maritimes et coloniales, t. 2, p. 726-731, 1820.

RENSEIGNEMENTS sur la fin de La Peyrouse. Bulletin Société Géographique de France, Paris, v. 7, p. 86-87, 1825.

RICHARDS, Rhys. Into the South Seas. The Southern Whale Fishery comes of Age on the Brazil Banks, 1765-1812. Wellington: The Paremata Press, 1994. ROUDER, Jane. Matthew Maury's Whale Maps: A Chapter in the History of Thematic Biological Cartography. Madison: University of Wisconsin, 1980. ROUSSEL DE VAUZEME, Augustin. Description du Cetochilus Australis, nouveau genre de Crustacé branchiopode. Annales des Sciences Naturelles, p. 333-338, 1834. 
SINEUX, Catherine. La Pêche de la baleine en France au $19^{\text {ème }}$ siècle illustrée par les campagnes des navires Constance (1830-1836) et Gange (1834-1845). Mémoire de Maitrise (Histoire) - Université de Paris-Sorbonne, Paris, 1986.

SMITH, Tim et al. Spatial and Seasonal Distribution of American Whaling and Whales in the Age of Sail. Plos One, v. 7, n. 4, 2012.

SCHOTTE, Margaret. Regimented Lessons: The Evolution of the Nautical Logbook in France. Annuaire de Droit Maritime et Océanique, v. 31, p. 91-115, juin 2013.

TILLMAN, Michael F; DONOVAN, Gregory P. (ed.). International Whaling Commission. Historical Whaling Records. Issue 5. Cambridge: [s. n.], 1983.

TOWNSEND, Charles H. The Distribution of Certain Whales as Shown by Logbook Records of American Whaleships. Zoologica, v. 19, p. 1-50, 1935. WEDDELL, James. A Voyage towards the South Pole: Performed in the Years 1822-24. Londres: Longman, Hurst, Rees, Orme, Brown, and Green, 1825. 